\title{
Perspectives franco-italiennes. Séminaires du CEFI 2000-2002. Etudes réunies et publiées par Luca Badini Confalonieri
}

Wilma Proglio

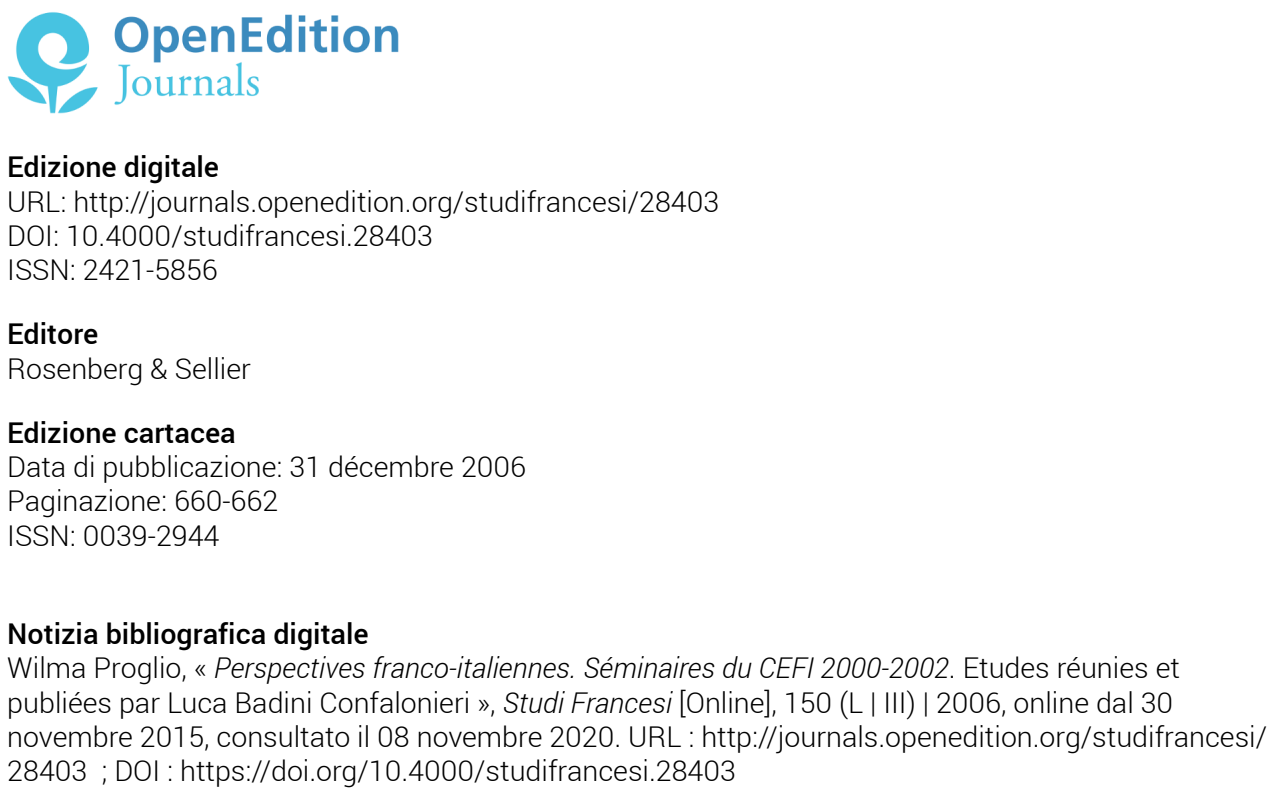

Questo documento è stato generato automaticamente il 8 novembre 2020.

\section{(ब) $\odot \Theta \Theta$}

Studi Francesi è distribuita con Licenza Creative Commons Attribuzione - Non commerciale - Non opere derivate 4.0 Internazionale. 


\title{
Perspectives franco-italiennes. Séminaires du CEFI 2000-2002. Etudes réunies et publiées par Luca Badini Confalonieri
}

\author{
Wilma Proglio
}

\section{NOTIZIA}

AA. VV., Perspectives franco-italiennes. Séminaires du CEFI 2000-2002. Etudes réunies et publiées par Luca BADINI CONFALONIERI, Roma, Aracne editrice, 2005, pp. 343.

1 In questo volume sono riuniti i contributi presentati durante le giornate seminariali organizzate dal Centre d'Etudes Franco-Italiennes e svoltesi a Chambéry tra il 2000 e il 2002. La prima parte del libro raccoglie interventi centrati sul tema del rapporto tra letteratura e mito. Alessandro BALLOR, nell'articolo Jason et Médée au Moyen Age (pp. 9-13), sottolinea come in quel perido tale mito sia stato sottoposto ad una duplice interpretazione, storica e morale. Dal punto di vista storico l'interesse dei letterati si è concentrato sul tema dell'avventura militare ed eroica degli Argonauti, mentre dal punto di vista morale l'attenzione si è spostata sulle drammatiche vicende dell'amore tra Giasone e Medea e dunque sui pericoli insiti nelle azioni dettate dalla passione. Nel contributo di Michele MASTROIANNI, Le monologue de Hémon dans l'Antigone" de Robert Garnier: traitement maniériste d'un mythe tragique classique (pp. 15-21), l'autore sottolinea come Garnier abbia preso le distanze dal testo sofocleo, assegnando, nella sua rivisitazione del mito, un interesse primario al tema dell'amore piuttosto che a quello della giustizia e della legge. Il cuore della pièce è dunque il tragico destino di una coppia di amanti infelici. Nell'intervento Il mito di Fedra nella rilettura operata dai Gesuiti (pp. 23-32), Marco LIVERA analizza la ripresa degli elementi e dei personaggi del mito antico nella tragedia cinquecentesca Crispus del padre gesuita Bernardino Stefonio. Al 
triangolo pagano Fedra-Ippolito-Teseo si sovrappone quello cristiano Fausta-CrispoCostantino. Nella messa in scena della lotta tra bene e male i gesuiti perseguono l'obiettivo di istruire e redimere i fedeli. Daniela DALLA VALLE, nell'articolo Appunti sul mito di Don Giovanni: le origini (pp. 33-41), mette in evidenza che gli elementi di fondo di questa vicenda (l'insulto al morto, la cena, il castigo finale) si possono riscontrare nel mito italiano di Leonzio, personaggio della cultura popolare, protagonista di canti e racconti di molte regioni del nostro paese. I tratti salienti del mito di Don Giovanni sono presenti anche in uno scenario della commedia dell'arte, dal titolo L'Ateista fulminato, il quale narra la storia di un conte di nome Aurelio. Dalla Valle sottolinea inoltre come la storia di Leonzio circolasse in ambienti gesuitici, in quanto edificante per il popolo. Nel contributo Récurrences de Pamela en France: Goldoni traduit et adapté (pp. 43-64), Lucia comparini analizza la figura della Pamela goldoniana, rivisitata nella letteratura francese del Settecento. Da simbolo della virtù ricompensata, mediante un matrimonio vantaggioso, Pamela diventa oggetto di riflessione psico-sociologica in merito all'opportunità o meno di infrangere le convenzioni sociali ascendendo a un rango più elevato del proprio in virtù del legame coniugale. L'ameno aspetto novellistico lascia dunque il posto ad una tradizione più arida e borghese della vicenda.

Nella seconda parte del volume sono raccolti gli articoli aventi per oggetto il rapporto tra letteratura e filosofia. Nell'intervento dal titolo L'invention de la philosophie chez Platon: imposture ou événement littèraire? (pp. 67-107), Pierre TORDJMAN sottolinea che l'«invenzione» della filosofia è di per sé un avvenimento letterario di prim'ordine. Platone, infatti, con i suoi scritti filosofici ha inaugurato un nuovo uso della lingua, privo di finalità pratica e oggettiva, volto esclusivamente al conseguimento della saggezza. Fondamentale nell'opera letteraria di Platone è inoltre il ricorso al mito e alla terminologia religiosa dei miti eleusini e del pitagorismo. Nel contributo intitolato $L a$ philosophie et le narrateur. La représentation du philosophe dans la nouvelle italienne du Moyen Age (pp. 109-122), Luca BADINI CONFALONIERI porta l'attenzione sul tema dell'immagine del filosofo, partendo da un aneddoto che vede per protagonista Talete, il quale cade in un pozzo poiché intento a contemplare le stelle mentre sta camminando e per la sua disattenzione viene deriso da una donna. Questo aneddoto può essere interpretato in modo ambivalente, sia come scherno del sapere astratto del filosofo, sia come critica della ristrettezza del pensiero popolare. Badini Confalonieri analizza inoltre una vita di Socrate pubblicata in una raccolta di biografie di personaggi illustri dell'antichità. In quel testo viene posto l'accento sull'aspetto fisico del filosofo, ritenuto sgradevole e ridicolo, e sulle sue controversie con le donne, le quali dunque sembrano fare spesso da contraltare ai pensatori, in virtù della loro mentalità concreta e pratica. Valentina MARTINI e Barbara MEAZZI sono autrici dell'articolo Il concetto di elasticità tra Bergson e Boccioni (pp. 123-137). Partendo dall'osservazione del dipinto futurista dal titolo Elasticità, le studiose indagano sulle occorrenze di questo termine in letteratura e in filosofia. Nel pensiero di Bergson il concetto di elasticità si identifica con la capacità dell'uomo di costruire significati attraverso l'intuizione, in una prospettiva temporale dilatata, scandita dagli stati di coscienza anziché dagli orologi. Nell'arte di Boccioni si trova dunque un'efficace rappresentazione del carattere proteiforme degli stati d'animo bergsoniani. Nell'intervento Moravia lecteur de Wittgenstein (pp. 139-153), Maryline MAIGRON approfondisce il debito che lo scrittore stesso dichiara di aver contratto dalla lettura del Tractatus logico-philosophicus del filosofo austriaco. 
3 La terza parte del libro è dedicata all'analisi del rapporto tra letteratura e politica. Nel contributo di Pierre tORDJMAN Littérature et politique: archéologie d'un rapport (pp. 157-167), l'autore risale all'autorità del poeta nell'antica Grecia e al valore quasi trascendente delle sue parole. Il poeta è dunque una sorta di mediatore tra i potenti e il popolo e tale ruolo ha la sua icona in Solone, poeta e legislatore. Antonella AMATUzzI, nell'articolo La littérature politique de la Fronde: une «guerre de plumes» au service de la langue française. L'exemple des Mazarinades du Cardinal de Retz (pp. 169-180), analizza dal punto di vista linguistico sette pamphlets di un esponente di spicco del movimento della Fronda. In essi il tono è aspramente polemico, è bandita ogni ricercatezza estetica ed abbondano gli stratagemmi retorici, finalizzati a persuadere il lettore. Le caratteristiche degli scritti prodotti in quel periodo ed in quel contesto politico sembrano aver contribuito al consolidarsi di una tradizione letteraria di impostazione rigorosa, che approderà al classicismo. Nell'intervento di Giovanni PAOLETTI, Le ambiguità della prospettiva: teoria politica e immaginazione letteraria in Necker e Constant (pp. 181-198), si prendono in considerazione alcune riflessioni politiche dei due scrittori, il primo favorevole al potere monarchico, il secondo invece di idee liberali. L'autore sottolinea poi come in Constant sia ricorrente su più livelli il tema del passaggio dall'illusione alla disillusione. Stefano BRUGNOLO, nel contributo La democrazia e la foresta: Tocqueville come viaggiatore (pp. 199-217), analizza il Voyage au lac Onéida, opera nella quale lo scrittore narra il cammino che egli intraprese nell'America settentrionale per andare sulle tracce di un nobile francese fuggito dopo la Rivoluzione. Il visitatore europeo si trova immerso nelle foreste, che evocano in lui il «mito rousseauiano della natura selvaggia», ma allo stesso tempo fanno sorgere nel suo animo un'inquietudine di sapore romantico. Nel caos che regna in questo mondo vegetale egli constata una sorta di parallelismo con la realtà sociale post-rivoluzionaria, nella quale è stato abolito l'ordine, un tempo garantito dalle gerarchie. Tocqueville assiste anche al taglio della foresta da parte dei conquistatori europei, giungendo a teorizzare il topos della violazione degli equilibri naturali come idillio interrotto, atto di prepotenza con il quale gli uomini vogliono piegare la natura ai loro piani e per il quale essi sono destinati comunque a pagare. Nell'articolo Un impegno politico e antiideologico (pp. 219-226), Dario voltolini riflette sul ruolo dello scrittore nel dibattito politico, ribadendo il pieno diritto del letterato alla libera espressione del proprio pensiero, espressione che deve però rimanere lontana dallo schieramento ideologico, in quanto lo scrittore mira a costruire significati, i quali possono scontrarsi con altri di segno opposto, senza avere tuttavia la pretesa di discernere in modo assoluto il vero dal falso.

4 La quarta ed ultima sezione del volume ha per oggetto il rapporto tra letteratura e religione. Nell'intervento Recherche et oubli de soi: Vittoria Colonna interprète de l'Evangile (pp. 229-242), Chiara PISACANE si sofferma sulla meditazione scritta dalla nobildonna italiana in merito alla parabola dell'adultera condannata alla lapidazione. Nel contributo di Franco PIPERNO, «L'ultimo è stato il Bruccioli...»: notes sur Antonio Brucioli et le rôle de la langue vulgaire (pp. 243-259), si porta l'attenzione sulla figura di questo umanista fiorentino, al quale si deve una traduzione della Bibbia in lingua volgare. A differenza di Lutero, che con la sua opera di traduzione gettò le basi della lingua tedesca, Brucioli non osò prendere troppa distanza dalle lingue tradizionalmente ritenute sacre, per questo la sua versione della Bibbia abbonda di elementi lessicali e sintattici calcati sull'ebraico e sul latino. Nell'articolo "Le Tragédies sainctes" di Louis de Masures: una proposta nel panorama delle tragedie bibliche (pp. 261-291), Luana PALLAGROSI 
analizza la trilogia cinquecentesca del poeta convertitosi alla riforma, trilogia costituita da David combatant, David triomphant e David fugitif. In queste tragedie ricorrono alcuni temi cari alla tradizione calvinista: la predestinazione, la miseria della condizione umana, il rapporto tra verità e illusione, la perseveranza nella fede e il rovescio della fortuna. Michele MASTROIANNI, nell'intervento dal titolo Quelques remarques sur l'emploi de la Bible dans le "Mespris de la vie et consolation contre la mort" de J.-B. Chassignet (pp. 293-299), analizza questo testo secentesco considerando la molteplicità di fonti a cui attinse il poeta. In una sorta di canzoniere sacro, composto da parafrasi bibliche, spicca la capacità di Chassignet di riscrivere i passi del testo sacro, imitando la maniera di alcuni suoi grandi predecessori. Nel contributo Letteratura e teatro nel primo Seicento francese: il teatro di Jean Prévost (pp. 301-311), Monica PAVESIO si sofferma sulle opere di questo scrittore, testimonianze del tentativo degli umanisti di conciliare fede cristiana e letteratura antica. In particolare la tragedia Hercule e la tragicommedia Clotilde narrano due vicende in cui l'antichità pagana non viene rinnegata, ma al contrario riscoperta e valorizzata, dopo esser stata riletta alla luce della fede cattolica. Nell'articolo di Simona MUNARI dal titolo Il conflitto religioso nel romanzo moresco francese del Seicento (pp. 313-331), l'attenzione si sofferma sull'approccio degli scrittori occidentali nei confronti dei personaggi di fede islamica. L'unica via loro concessa per evolversi è la conversione, la quale tuttavia può avvenire solo se sussistono determinati requisiti. Il moro, infatti, deve essere nobile d'animo e di natali, essere un guerriero coraggioso e conoscere le regole della cavalleria e deve inoltre poter vantare un'antica ascendenza cristiana.

5 In questo volume si alternano l'uso della lingua francese ed italiana, in piena osservamza dello spirito delle giornate seminariali dalle quali è scaturita tale opera, vale a dire la volontà di dimostrare l'importanza del confronto e del dialogo tra le due letterature, geograficamente contigue, ma caratterizzate ciascuna da elementi originali e specifici. 